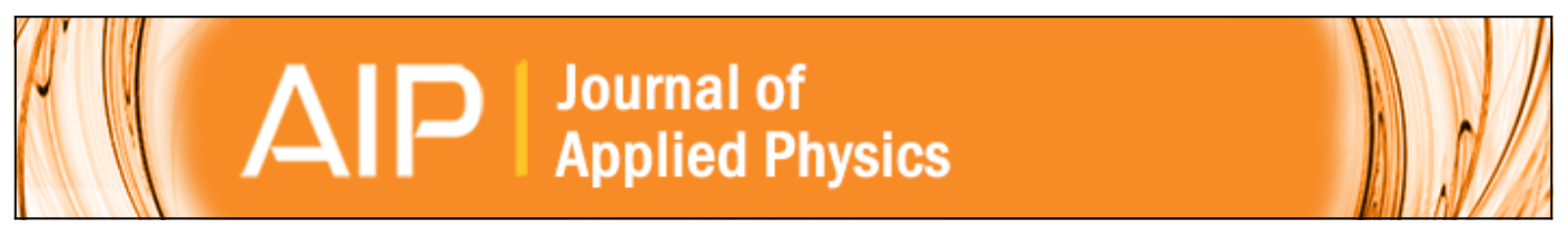

\title{
Enhanced sensitivity to direct current magnetic field changes in Metglas/Pb(Mg1/3Nb2/3)O3-PbTiO3 laminates
}

Junqi Gao, Liangguo Shen, Yaojing Wang, David Gray, Jiefang Li, and D. Viehland

Citation: Journal of Applied Physics 109, 074507 (2011); doi: 10.1063/1.3569629

View online: http://dx.doi.org/10.1063/1.3569629

View Table of Contents: http://scitation.aip.org/content/aip/journal/jap/109/7?ver=pdfcov

Published by the AIP Publishing

\section{Articles you may be interested in}

Magnetoelectric effect of the multi-push-pull mode in $0.35 \mathrm{~Pb}(\ln 1 / 2 \mathrm{Nb} 1 / 2) \mathrm{O} 3-0.35 \mathrm{~Pb}(\mathrm{Mg} 1 / 3 \mathrm{Nb} 2 / 3) \mathrm{O} 3-$

0.30PbTiO3/Metglas magnetoelectric composite

J. Appl. Phys. 114, 027011 (2013); 10.1063/1.4812221

Overlap of the intrinsic and extrinsic magnetoelectric effects in BiFeO3-PbTiO3 compounds: Potentialities for magnetic-sensing applications

J. Appl. Phys. 113, 034102 (2013); 10.1063/1.4775796

An improved magnetic field detection unit based on length-magnetized Terfenol-D and width-polarized ternary $0.35 \mathrm{~Pb}(\operatorname{In} 1 / 2 \mathrm{Nb} 1 / 2) \mathrm{O} 3-0.35 \mathrm{~Pb}(\mathrm{Mg} 1 / 3 \mathrm{Nb} 2 / 3) \mathrm{O} 3-0.30 \mathrm{PbTiO} 3$

Appl. Phys. Lett. 101, 232906 (2012); 10.1063/1.4769904

Large converse magnetoelectric effect in Metglas FeCoBSi and $0.7 \mathrm{~Pb}$ ( Mg 1 / 3 Nb 2 / 3 ) O 3 - 0.3 PbTiO 3 laminated composite

Appl. Phys. Lett. 98, 052505 (2011); 10.1063/1.3549871

Ultrahigh magnetic field sensitivity in laminates of TERFENOL-D and $\mathrm{Pb}(\mathrm{Mg} 1 / 3 \mathrm{Nb} 2 / 3$ ) O $3-\mathrm{PbTiO} 3$ crystals Appl. Phys. Lett. 83, 2265 (2003); 10.1063/1.1611276

MIT LINCOLN

LABORATORY CAREERS

Discover the satisfaction of innovation and service

to the nation
- Space Control

- Air \& Missile Defense

- Communications Systems \& Cyber Security

- Intelligence, Surveillance and

Reconnaissance Systems

\section{LINCOLN LABORATORY}

Massachusetts Institute of TechNology

- Advanced
Electronics
- Tactical Systems
- Homeland
Protection
- Air Traffic Control

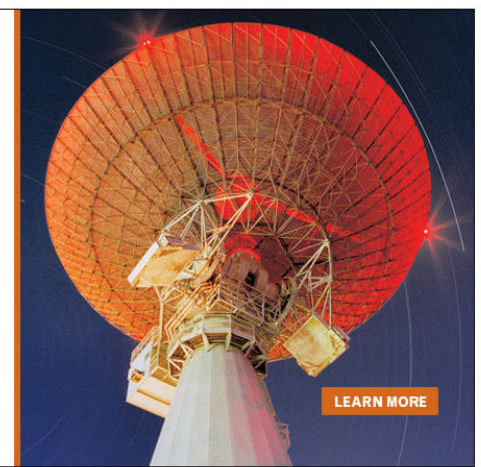




\title{
Enhanced sensitivity to direct current magnetic field changes in Metglas/ $\mathrm{Pb}\left(\mathrm{Mg}_{1 / 3} \mathrm{Nb}_{2 / 3}\right) \mathrm{O}_{3}-\mathrm{PbTiO}_{3}$ laminates
}

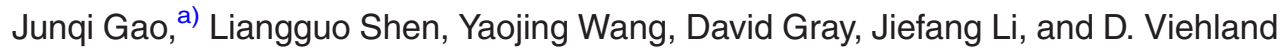 \\ Department of Materials Science and Engineering, Virginia Tech, Blacksburg, Virginia 24061, USA
}

(Received 22 December 2010; accepted 21 February 2011; published online 7 April 2011)

\begin{abstract}
We have developed Metglas/ $\mathrm{Pb}\left(\mathrm{Mg}_{1 / 3} \mathrm{Nb}_{2 / 3}\right) \mathrm{O}_{3}-\mathrm{PbTiO}_{3}$ magnetoelectric (ME) laminates that have notably larger ME coefficients, with maximum values of up to $45 \mathrm{~V} / \mathrm{cm}$ Oe. Based on this giant ME effect, the dc magnetic field sensitivity for Metglas/PMN-PT laminate sensors was improved by a factor of $>3$, relative to that for Metglas $/ \mathrm{Pb}(\mathrm{Zr}, \mathrm{Ti}) \mathrm{O}_{3}$ (PZT)-based ones of the same geometry. Our new ME sensor can detect dc magnetic field changes as small as (i) $5 \mathrm{nT}$ at $1 \mathrm{kHz}$ and (ii) $1 \mathrm{nT}$ near the resonant frequency in a shield chamber. (C) 2011 American Institute of Physics. [doi:10.1063/1.3569629]
\end{abstract}

\section{INTRODUCTION}

There are many types of magnetic sensors, including giant magnetoresistive (GMR), flux-gate, and superconducting quantum interference devices (SQUID). They have low noise floors on the order of $10^{-10}, 10^{-12}$, and $10^{-14} \mathrm{~T} / \sqrt{\mathrm{Hz}}$, respectively in the frequency range of $1<f<10^{3} \mathrm{~Hz}^{1-3}$ Such low noise floors offer the opportunity to develop highly sensitive magnetometers. However, there are some limitations to these conventional magnetic sensors in applications. For example, SQUIDs require extremely low operational temperatures, fluxgates have magnetic hysteresis and offset values under zero magnetic fields, and both flux gate and GMRs require considerable power.

An alternative type of magnetic sensor has recently been developed based on a giant magnetoelectric (ME) effect. ${ }^{4}$ The ME effect was first observed about 50 years ago in $\mathrm{Cr}_{2} \mathrm{O}_{3}$ single crystals, which had a ME voltage coefficient of $\alpha_{\mathrm{ME}} \approx 20 \mathrm{mV} / \mathrm{cm}$ Oe. ${ }^{5}$ Since 2001 , ME laminated composites have been found that have ME voltage coefficients several orders of magnitude higher than that of single phase materials. ${ }^{6}$ Long-type sandwiched laminate structures comprised of magnetostrictive Metglas and piezoelectric $\mathrm{Pb}(\mathrm{Zr}, \mathrm{Ti}) \mathrm{O}_{3}$ or PZT layers have values of $\alpha_{\mathrm{ME}} \leq 22 \mathrm{~V} / \mathrm{cm} \mathrm{Oe}^{7}$ Room temperature, passive, and highly sensitive magnetic sensors have been developed based on these ME heterostructures. In addition, geomagnetic sensors based on these Metglas/ PZT-fiber laminates ${ }^{8}$ have been shown capable of sensing earth's field and inclination, and detecting changes there within.

Dong et $a l .^{9,10}$ developed an equivalent method by which to understand the ME coefficients of heterostructural composites. Following this method, $\alpha_{\mathrm{ME}}$ should be linearly proportional to the piezoelectric voltage coefficient $g_{33, p}$, and a function of the piezoelectric coupling coefficient $k_{33, p}$. Here, we have developed multi-push-pull longitudinal-longitudinal or $\mathrm{L}-\mathrm{L}$ mode Metglas/ $\mathrm{Pb}\left(\mathrm{Mg}_{1 / 3} \mathrm{Nb}_{2 / 3}\right) \mathrm{O}_{3}-\mathrm{PbTiO}_{3}$ (PMN-PT) single crystal fiber ME laminates. Due to higher

\footnotetext{
a) Author to whom correspondence should be addressed. Electronic mail: junqi08@vt.edu.
}

values of $g_{33, p}$ and $k_{33, p}$, the maximum value of $\alpha_{\mathrm{ME}}$ for these sensors is about a factor of 3 higher than that for Metglas/ PZT laminates of similar geometry. This enhancement in $\alpha_{\mathrm{ME}}$ results in a three times increase in dc magnetic field sensitivity when driven in an active mode: enabling detection of dc magnetic field changes of $H_{\mathrm{dc}} \leq 1 \mathrm{nT}$.

\section{EXPERIMENTAL DETAILS}

To fabricate Metglas/PZT and Metglas/PMN-PT laminates, we obtained PZT (Smart Materials, Sarasota, FL) and PMN-PT (Ceracomp Co., Ltd., Korea) fibers, and Metglas foils (Vitrovac Inc., Hanau, German). The piezoelectric properties for these PZT and PMN-PT fibers are given in Table I. Five pieces of piezoelectric fibers of $180 \mu \mathrm{m}$ in thickness were oriented along the long axes to form a layer that was $10 \mathrm{~mm}$ wide and $40 \mathrm{~mm}$ long. Two interdigitated Kapton-based electrodes were then bonded to the top and bottom surfaces of the piezoelectric layer in a multi-pushpull geometry. Three Metglas foils of $80 \mathrm{~mm}$ in length and $10 \mathrm{~mm}$ in width were then laminated to both the top and bottom surfaces of the piezoelectric layer, in order to achieve the optimized volume ratio between piezoelectric and magnetoelectric phases. ${ }^{11}$ Details of the laminate fabrication method can be found in Ref. 7.

\section{RESULTS AND DISCUSSION}

First, the ME voltage coefficient $\alpha_{\mathrm{ME}}$ was measured as a function of dc magnetic field $H_{\mathrm{dc}}$ for the different ME laminates. In this test, a lock-in amplifier (SR-850) was used to apply the driving signal to a pair of Helmholtz coils, which generated an ac magnetic field of $H_{\mathrm{ac}}=1$ Oe at frequency of $f=1 \mathrm{kHz}$. A dc magnetic field $H_{\mathrm{dc}}$ was then applied along the long axis of the ME laminates using permanent magnets. Figure 1(a) shows $\alpha_{\mathrm{ME}}$ as a function of $H_{\mathrm{dc}}$ for Metglas/ PMN-PT and Metglas/PZT laminates. In Fig. 1(a), we can see that $\alpha_{\mathrm{ME}}$ for the two ME laminates had similar trends with $H_{\mathrm{dc}}$; however, the values of $\alpha_{\mathrm{ME}}$ for the Metglas/PMNPT laminate was notably higher than that for the Metglas/ PZT one. In particular, the maximum value of $\alpha_{\mathrm{ME}}$ for the Metglas/PMN-PT laminate was $45 \mathrm{~V} / \mathrm{cm} \mathrm{Oe,} \mathrm{which} \mathrm{was}$ 
TABLE I. The critical piezoelectric properties for PZT and PMN-PT fibers.

\begin{tabular}{lccc}
\hline \hline & $d_{33, p}(\mathrm{pC} / \mathrm{N})$ & $g_{33, p}(\mathrm{mV} \mathrm{m} / \mathrm{N})$ & $k_{33}$ \\
\hline PZT $^{\mathrm{a}}$ & 440 & 25.5 & 0.72 \\
PMN-PT $^{\mathrm{b}}$ & 2000 & 32.4 & 0.93 \\
\hline \hline
\end{tabular}

${ }^{\mathrm{a} C}$ Cited from Smart Material Corp., USA.

${ }^{\mathrm{b}}$ Cited from Ceracomp Co., Ltd., Korea.

about three times larger than that for the PZT based one of similar size (i.e., $15 \mathrm{~V} / \mathrm{cm} \mathrm{Oe}$ ). This is the highest value of $\alpha_{\mathrm{ME}}$ reported to date for any ME composite, at least by a factor of 2.

Figure 1(b) shows the ME voltage coefficient for Metglas/PZT and Metglas/PMN-PT laminates as a function of ac magnetic field frequency, while sweeping through the electromechanical resonance (EMR). The fundamental resonant frequencies for the PZT and PMN-PT based sensors were 31.5 and $27.8 \mathrm{kHz}$, respectively. In Fig. 1(b), we can see (i) a strong EMR enhancement in $\alpha_{\mathrm{ME}}$ that has previously been reported $^{7}$ and (ii) that values of $\alpha_{\mathrm{ME}}>1100 \mathrm{~V} / \mathrm{cm} \mathrm{Oe}$ can be reached for PMN-PT laminates, which was about $3 \times$ larger than that for PZT ones.

Next, the dc magnetic field sensitivity was characterized for both sensors using an active method: ${ }^{12}$ a 200-turn coil was wrapped around the sensor that carried a small ac
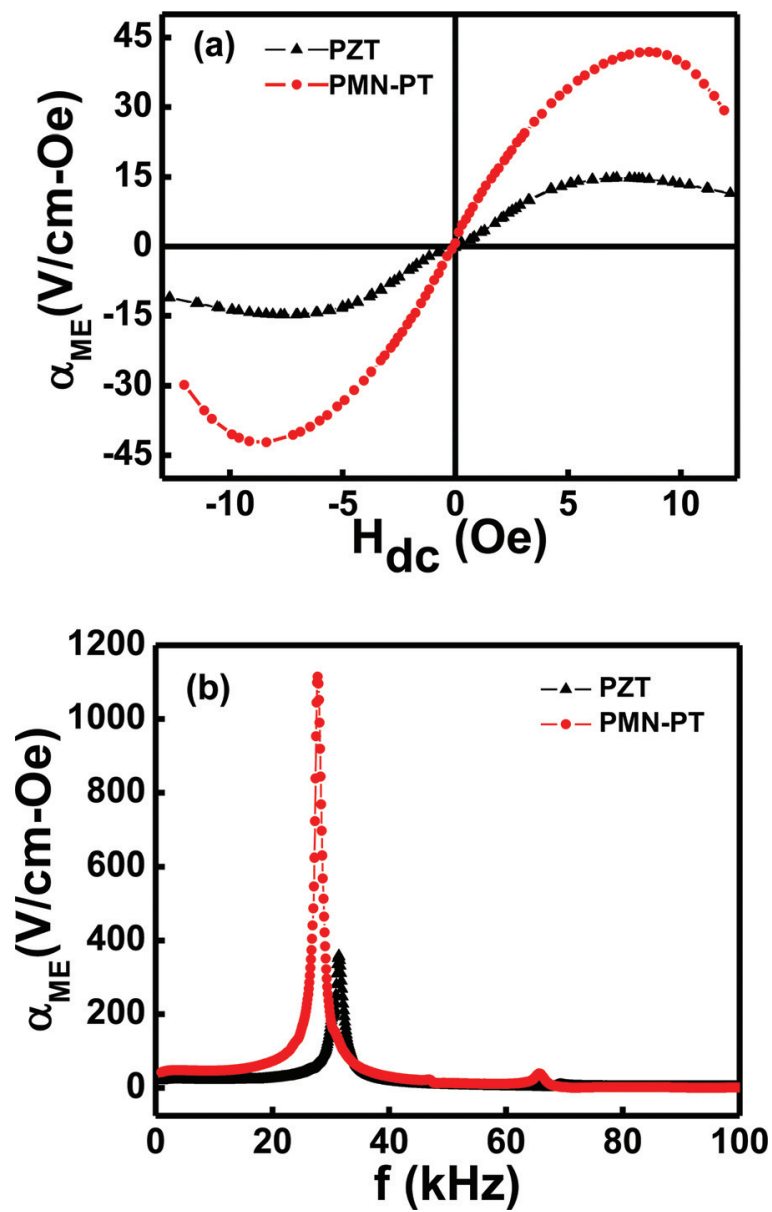

FIG. 1. (Color online) ME voltage coefficient of Metglas/PZT and Metglas/ PMN-PT laminates: (a) $\alpha_{\mathrm{ME}}$ as the function of dc bias $H_{\mathrm{dc}}$ at $f=1 \mathrm{kHz}$, and (b) $\alpha_{\mathrm{ME}}$ as a function of ac magnetic drive frequency. current provided by the lock-in amplifier to drive the $\mathrm{ME}$ sensors. Voltages were then induced in the piezoelectric layer by small changes in $H_{\mathrm{dc}}$, which were measured by the amplifier. In all of these measurements, we kept the signalto-noise ratio constant at $\mathrm{SNR}=10$. Figures 2 (a) and 2(b) show the induced output voltages from the Metglas/PZT laminates in response to small changes in $H_{\mathrm{dc}}$ at driving frequencies of $f=1$ and $10 \mathrm{kHz}$, respectively. It can be seen that the dc magnetic field variations as small as $H_{\mathrm{dc}}=15 \mathrm{nT}$ can be detected for $f=1 \mathrm{kHz}$ and $H_{a c}=0.1$ Oe. Even higher sensitivity to small changes in $H_{\mathrm{dc}}$ were found for $f=10 \mathrm{kHz}$ and $H_{a c}=0.1$ Oe: where changes as low as $12 \mathrm{nT}$ could be detected at a constant $\mathrm{SNR}=10$.

Figures 3(a) and 3(b) show similar sensitivity measurements to small changes in $H_{\mathrm{dc}}$ at a constant $\mathrm{SNR}=10$ for Metglas/PMN-PT laminates. In Fig. 3, one can see that the sensitivity for the Metglas/PMN-PT laminates was significantly enhanced relative to that for the Metglas/PZT ones. In Fig. 3(a), the sensitivity to dc magnetic field changes for PMN-PT laminates can be seen to be $5 \mathrm{nT}$ at $1 \mathrm{kHz}$ and $H_{\mathrm{ac}}=0.1$ Oe: which was three times higher than that for PZT based ones with a constant SNR. Further, as can be seen in Fig. 3(b), the sensitivity at $f=10 \mathrm{kHz}$ can also be seen to be $3 \times$ higher for Metglas/PMN-PT than Metglas/PZT. This enhancement in dc field sensitivity is a direct consequence of the higher values of $\alpha_{\mathrm{ME}}$ for the Metglas/PMN-PT laminates. Please note that the value of $\left|\partial \alpha_{\mathrm{ME}} / \partial H_{\mathrm{dc}}\right|$ was also much larger for Metglas/PMN-PT than Metglas/PZT, over the range of $-5 \mathrm{Oe}<H_{\mathrm{dc}}<5 \mathrm{Oe}$, as can be seen in Fig. 1(a).

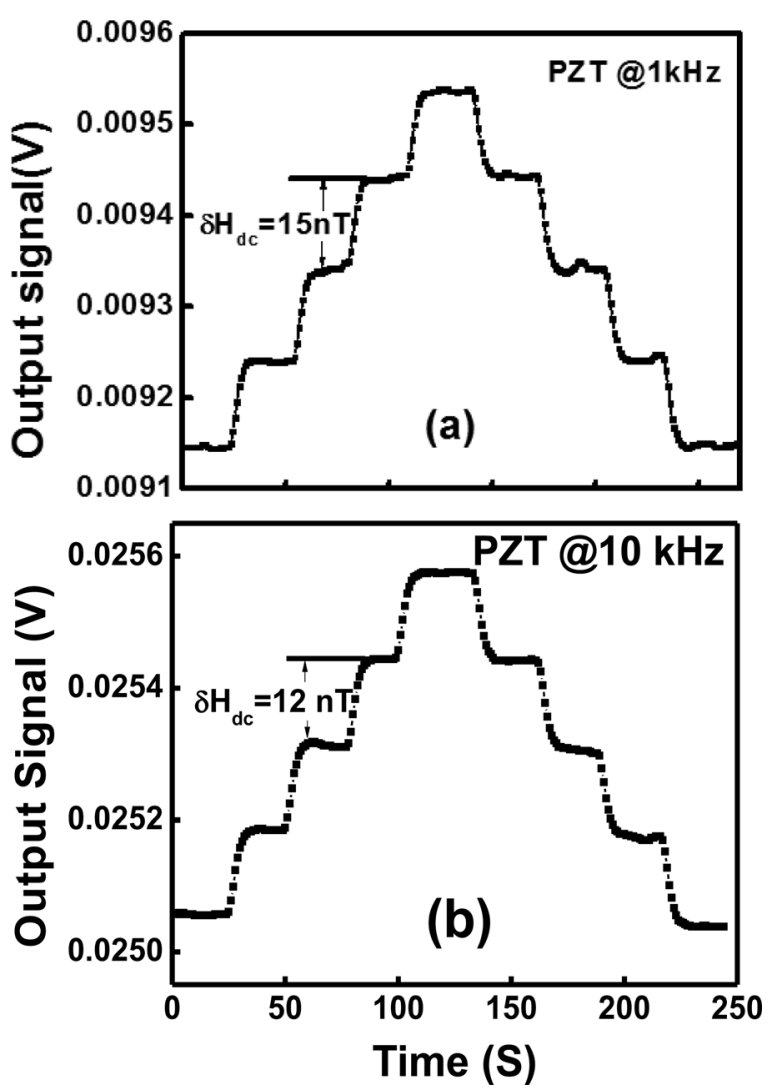

FIG. 2. Sensitivity of Metglas/PZT laminate to small dc magnetic field changes under ac drive conditions of: (a) at $f=1 \mathrm{kHz}$ and $H_{\mathrm{ac}}=0.1 \mathrm{Oe}$, and (b) at $f=10 \mathrm{kHz}$ and $H_{\mathrm{ac}}=0.1 \mathrm{Oe}$. 

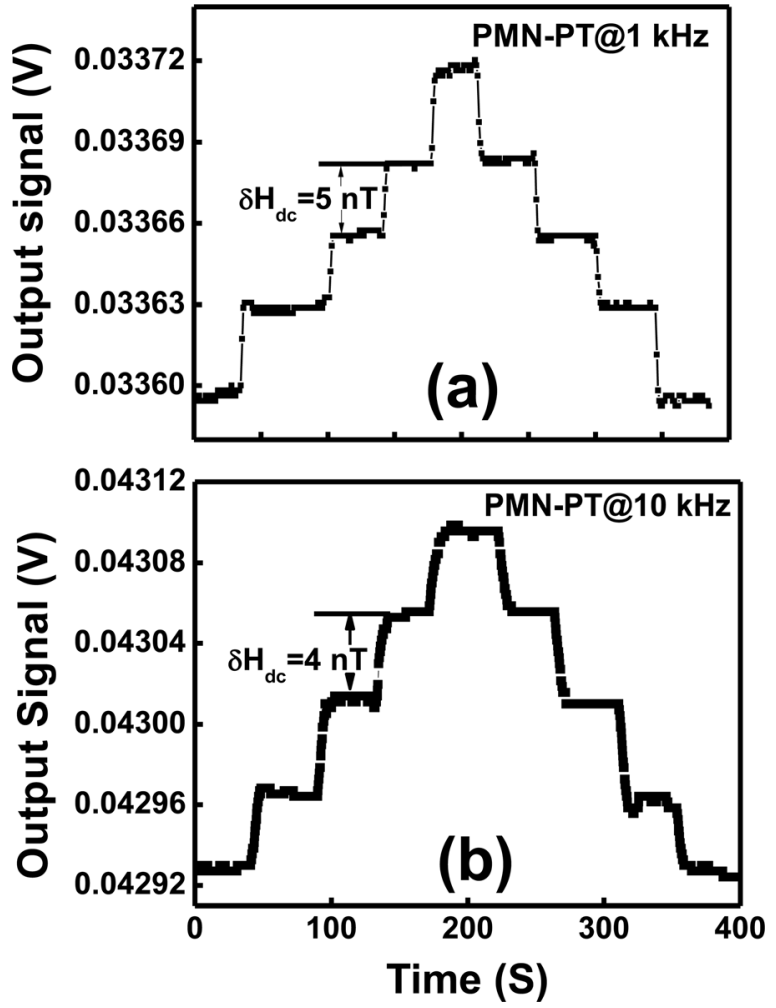

FIG. 3. Sensitivity of Metglas/PMN-PT laminate to small dc magnetic field changes under ac drive conditions of: (a) at $f=1 \mathrm{kHz}$ and $H_{\mathrm{ac}}=0.1 \mathrm{Oe}$, and (b) at $f=10 \mathrm{kHz}$ and $H_{\mathrm{ac}}=0.1 \mathrm{Oe}$.

Finally, the sensitivity to small dc magnetic field changes for Metglas/PMN-PT laminates was studied under the EMR conditions $(f=27.8 \mathrm{kHz})$. As $\alpha_{\mathrm{ME}}$ is extremely high in this case [see Fig. 1 (b)], the ME sensor was placed in a magnetically shielded chamber to reduce exposure to environmental noise. Figure 4 shows the induced output voltage to small step changes in a dc magnetic field. Clearly, the Metglas/PMN-PT laminates can detect changes of $H_{\mathrm{dc}} \leq 1$ $\mathrm{nT}$. This represents a notable improvement in dc field sensitivity relative to lower frequencies using the same laminates $(4-5 \times)$, to PZT-based laminates $(12-15 \times)$, and to prior reports $(\text { about } 10 \times)^{12}$

These dc field sensors might be used for a navigation based on variations in Earth's field, that is given an accurate database of $H_{\text {earth }}(x, y, z ; t)$ such as tabulated by the National Geophysical Data Center. ${ }^{13}$ Some migratory animals are believed to use a biological detection method for $H_{\text {earth }}$ to assist them, in conjunction with other cues, in their navigation. ${ }^{14,15}$ In consideration the average change in the variation of $H_{\text {earth }}$ is $0.02 \mathrm{nT} / \mathrm{m}$ (Ref. 13), the dc field sensitivity of $\leq 1$ $\mathrm{nT}$ for our ME sensor could offer the potential of localization or navigation with a precision of $50 \mathrm{~m}$.

\section{SUMMARY}

We have realized a significant enhancement in $\alpha_{\mathrm{ME}}$ (by $3 \times$ ) and the dc magnetic field sensitivity (by up to $10 \times$ ) by

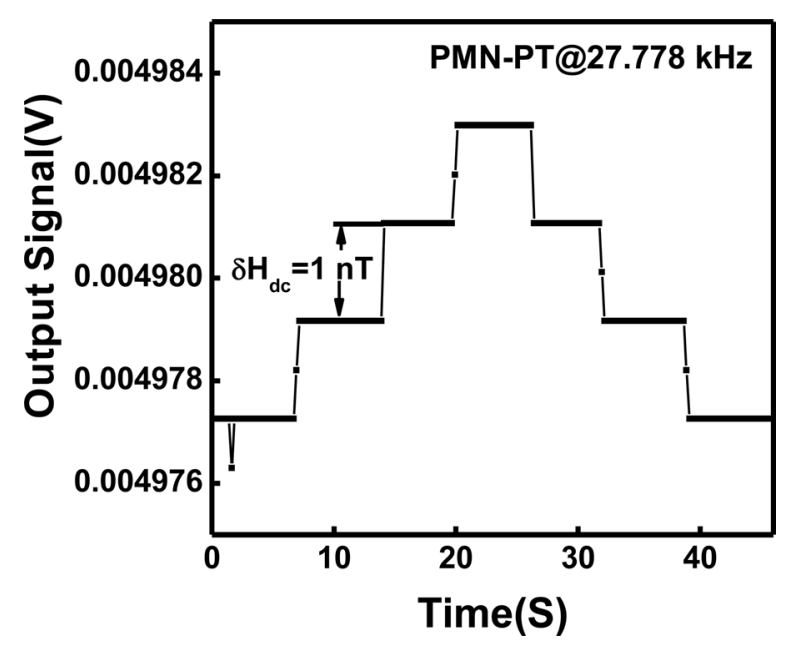

FIG. 4. Sensitivity of Metglas/PMN-PT laminate to small dc magnetic field changes under ac drive field of $H_{\mathrm{ac}}=0.1 \mathrm{Oe}$ at the resonant frequency.

using PMN-PT fibers in heterostructured composites, relative to the values of PZT fibers. The ME voltage coefficient for Metglas/PMN-PT laminates reached values of $45 \mathrm{~V} / \mathrm{cm}$ Oe at $f=1 \mathrm{kHz}$, and of $1100 \mathrm{~V} / \mathrm{cm}$ Oe at the EMR. The dc field sensitivity of these Metglas/PMN-PT laminates was then found to be $4 \mathrm{nT}$ under a constant drive of $H_{\mathrm{ac}}=0.1$ Oe at $f=10$ $\mathrm{kHz}$. Even smaller dc field changes of $\leq 1 \mathrm{nT}$ were also detected at the EMR. Such high sensitivities to small dc field changes have the potential for localization or navigation based on variations in the geomagnetic field.

\section{ACKNOWLEDGMENTS}

The authors would like to thank DARPA and ONR for support of this work.

${ }^{1}$ P. Eitenne, G. Creuzet, A. Friederich, and J. Chazelas, Phys. Rev. Lett. 61, 2472 (1988).

${ }^{2}$ K. Shirae, IEEE Trans. Magn. 20, 1299 (1984).

${ }^{3}$ H. Bartehelmess, M. Halverscheid, B. Schiefenhovel, E. Heim, M. Schilling, and R. Zimmermann, IEEE Trans. Appl. Supercond. 11, 657 (2001).

${ }^{4}$ J. Y. Zhai, S. X. Dong, J.-F. Li, and D. Viehland, Appl. Phys. Lett. 88, 062510 (2006).

${ }^{5}$ G. T. Rado and V. J. Folen, Phys. Rev. Lett. 7, 310 (1961).

${ }^{6}$ C-W. Nan, M. I. Bichurin, S. Dong, D. Viehland, and G. Srinivasan, J. Appl. Phys. 103, 031101 (2008).

${ }^{7}$ S. X. Dong, J. Y. Zhai, J.-F. Li, and D. Viehland, Appl. Phys. Lett. 89, 252904 (2006).

${ }^{8}$ J. Y. Zhai, S. X. Dong, Z. P. Xing, J.-F. Li, and D. Viehland, Appl. Phys. Lett. 91, 123513 (2007).

${ }^{9}$ S. X. Dong, J. F. Li, and D. Viehland, IEEE Trans. Ultrason. Ferroelectr. Freq. Control. 50, 1253 (2003).

${ }^{10}$ S. X. Dong, J. F. Li, and D. Viehland, J. Mater. Sci. 41, 97 (2006).

${ }^{11}$ J. Das, J. Gao, Z. Xing, J. F. Li, and D. Viehland, Appl. Phys. Lett. 95, 092501 (2009).

${ }^{12}$ S. X. Dong, J. Y. Zhai, J.-F. Li, and D. Viehland, Appl. Phys. Lett. 88, 082907 (2006).

${ }^{13} \mathrm{http}: / /$ www.ngdc.noaa.gov/geomag/.

${ }^{14}$ W. Cochran, H. Mouritsen, and M. Wikelski, Science 304, 405 (2004).

${ }^{15}$ K. J. Lohmann, C. M. F. Lohmann, L. M. Ehrhart, D. A. Bagley, and T. Swing, Nature 428, 909 (2004). 